\title{
ADDITIVE REPRESENTATION IN THIN SEQUENCES, V: MIXED PROBLEMS OF WARING'S TYPE
}

\author{
J. BRÜDERN, K. KAWADA and T. D. WOOLEY*
}

\section{Introduction}

In the first part of this series of papers (see Brüdern, Kawada and Wooley [2]), we introduced an approach to additive problems in which one seeks to establish that almost all natural numbers in some fixed polynomial sequence are represented in a prescribed manner, thereby deriving non-trivial estimates for exceptional sets in thin sequences. We illustrated our methods by obtaining upper bounds for the exceptional sets associated with the representation of integers from quadratic, or cubic, polynomial sequences by sums of six cubes of positive integers. In subsequent parts of the series (see Brüdern, Kawada and Wooley [3], [4], [5]), we adapted our core methods so as to tackle problems associated with the binary Goldbach problem, the expected asymptotic formula for the number of representations, and lower bounds for the number of integers represented in some prescribed manner. As is apparent from the opening part of this series, our methods are of great flexibility. The aim of the present paper is to provide variants of the ideas developed in the preceding opera, and here we will be concerned solely with methods which provide estimates for the size of exceptional sets in representation problems. The discerning reader will recognise that in several of the more exotic problems mentioned below, it is the existence of a non-trivial estimate for the exceptional set in question which is of interest. The investigation of the sharpest attainable estimate for this exceptional set should be politely deferred beyond any future occasion.

We begin by exploring exceptional sets in polynomial sequences for additive problems involving mixed powers. Here one finds that sharp mean value estimates for mixed sums of powers, familiar to aficionados of the circle method, lead to surprisingly strong conclusions. Our first results, which we establish in $\$ 2$, involve problems containing a block of four cubes. Here and elsewhere,

\footnotetext{
* Packard Fellow, and supported in part by NSF grant DMS-9622773. This paper benefitted from visits of various of the authors to Ann Arbor, Kyoto, Oberwolfach and Stuttgart, and the authors collectively thank these institutions for their hospitality and excellent working conditions.

Received August 16, 2000.
} 
it is convenient to describe a polynomial $\phi \in \mathrm{Q}[t]$ as being an integral polynomial if, whenever the parameter $t$ is an integer, then the value $\phi(t)$ is also an integer.

THEOREM 1.1. Suppose that $\phi$ is an integral quadratic polynomial with positive leading coefficient. Denote by $E_{\phi}(X)$ the number of integers $n$ with $1 \leq n \leq X$ such that $\phi(n)$ possesses no representation as the sum of five cubes and a sixth power of positive integers. Then for each positive number $\varepsilon$, one has $E_{\phi}(X) \ll X^{37 / 42+\varepsilon}$. Here, the implicit constant in Vinogradov's notation may depend on $\phi$ and $\varepsilon$.

In Theorem 1.1 of Brüdern, Kawada and Wooley [2], we established that almost all values of a fixed integral quadratic polynomial, with positive leading coefficient, are the sum of six cubes of positive integers. As an immediate consequence of Theorem 1.1 above, we find that one may specialise one of the latter cubes to be a sixth power, and nonetheless derive a similar conclusion. For the purposes of illustration, in the following theorem we record several further applications of estimates involving the block of four cubes.

THEOREM 1.2. (a) Let $\phi_{2}$ be an integral quadratic polynomial with positive leading coefficient. Then for almost all natural numbers $n$, one has that $\phi_{2}(n)$ is the sum of any one of the following combinations of powers:

(i) five cubes and a $k$-th power, for $3 \leq k \leq 20$;

(ii) four cubes and two biquadrates;

(iii) four cubes and two fifth powers.

(b) Let $\phi_{3}$ be an integral cubic polynomial with positive leading coefficient. Then for almost all $n$, one has that $\phi_{3}(n)$ is the sum of a square, four cubes and a sixth power.

We remark that in part (i) of Theorem 1.2 (a), the permissible range for $k$ may certainly be considerably extended with greater effort, and indeed our calculations indicate that values of $k$ in the mid-forties are permissible. We note also that explicit estimates for the exceptional sets implicit in the statement of Theorem 1.2 may be inferred from the concluding display of the proof of Theorem 1.2 in $\$ 2$ below. We do not claim, however, that these estimates are close to the best attainable via modern technology.

It is possible to modify the argument underlying the proofs of Theorems 1.1 and 1.2 so as to replace the block of four cubes by a new block of three cubes, together with a fourth, fifth or sixth power. Since the situation with three cubes and a sixth power is the most difficult, we illustrate our ideas with this case and leave the reader to fill in the necessary details for the easier cases in which the sixth power is replaced by a fourth or fifth power. In $\S 3$ we establish the 
(somewhat exotic) conclusions contained in the following theorem. We caution the reader that our aim here is mostly to illustrate ideas.

THEOREM 1.3. (a) Let $\phi_{2}$ be an integral quadratic polynomial with positive leading coefficient. Then for almost all natural numbers $n$, one has that $\phi_{2}(n)$ is the sum of either of the following combinations of powers:

(i) four cubes and two sixth powers;

(ii) three cubes, two biquadrates and a sixth power.

(b) Let $\phi_{3}$ be an integral cubic polynomial with positive leading coefficient. Then, for almost all $n$, one has that $\phi_{3}(n)$ is the sum of a square, three cubes and two sixth powers.

(c) Let $\phi_{4}$ be an integral quartic polynomial with positive leading coefficient. Then, for almost all $n$, one has that $\phi_{4}(n)$ is the sum of a square, three cubes, a biquadrate and a sixth power.

(d) Let $\phi_{6}$ be an integral sextic polynomial with positive leading coefficient. Then, for almost all $n$, one has that $\phi_{6}(n)$ is the sum of a square, four cubes and a sixth power.

A rather different strategy may be adopted in certain problems involving squares. We illustrate such ideas in $\$ 4$ by considering values of cubic polynomials represented as the sum of a square and four cubes of positive integers.

THEOREM 1.4. Let $\phi_{3}$ be an integral cubic polynomial with positive leading coefficient. Then, for almost all natural numbers $n$, one has that $\phi_{3}(n)$ is the sum of a square and four cubes of positive integers.

Next we consider polynomial sequences represented by sums of $k$-th powers, for larger $k$. When $\phi$ is an integral polynomial with positive leading coefficient, denote by $G_{\phi}^{+}(k)$ the least number $s$ with the property that for almost all natural numbers $n$, one has a representation of $\phi(n)$ in the shape

$$
x_{1}^{k}+x_{2}^{k}+\ldots+x_{s}^{k}=\phi(n),
$$

with $x_{i} \in \mathrm{N}(1 \leq i \leq s)$. Also, when $d$ is a natural number, define $G_{d}^{+}(k)$ to be the supremum, taken over all integral polynomials $\phi$ of degree $d$ with positive leading coefficient, of $G_{\phi}^{+}(k)$. In $\S 5$ we consider the representation of polynomial sequences of degree $d \geq 2$, and in $\$ 6$ we refine the associated estimates in the quadratic case. Taken together, our conclusions may be summarised as follows.

TheOREM 1.5. Suppose that $d=1$ or 2. Then for large $k$ one has

$$
G_{d}^{+}(k) \leq \frac{1}{2} k\left(\log k+\log \log k+c_{d}+o(1)\right),
$$


where

$$
c_{d}= \begin{cases}2, & \text { when } d=1 \\ \frac{5}{2}+\log 2, & \text { when } d=2\end{cases}
$$

When $d>2$, on the other hand, one has

$$
G_{d}^{+}(k) \leq(1-1 / d) k(\log k+\log \log k+O(1)) .
$$

We remark that when $d=1$, the conclusion of Theorem 1.5 follows immediately from the proof of Theorem 1.4 of Wooley [20] (or see (6.2) and (6.3) below) via the standard method, and thus we confine our proof of Theorem 1.5 to the cases in which $d \geq 2$. It is curious that the number of variables required almost always to obtain a representation in the shape (1.1) is almost the same, in the current state of knowledge, for $\phi$ of degree both 1 and 2. For quadratic polynomials $\phi$, the work of Vaughan and Wooley [14], [15], [16], [17] may be utilised within the methods of $\S 6$ so as to obtain reasonable explicit estimates for $G_{\phi}^{+}(k)$.

THEOREM 1.6. When $3 \leq k \leq 20$, one has $G_{2}^{+}(k) \leq H_{2}(k)$, where $H_{2}(k)$ denotes the value presented in the table below.

We note that the bound for $G_{2}^{+}(3)$ presented here follows from Theorem 1.1 of Brüdern, Kawada and Wooley [2]. We have taken the liberty of recording in the table also values of $H_{1}(k)$ for which $G_{1}^{+}(k) \leq H_{1}(k)$. These additional estimates follow from the classical theory of Waring's problem.

\begin{tabular}{|rccccccccc|}
\hline$k$ & 3 & 4 & 5 & 6 & 7 & 8 & 9 & 10 & 11 \\
$H_{1}(k)$ & 4 & 15 & 9 & 12 & 17 & 32 & 25 & 30 & 34 \\
$H_{2}(k)$ & 6 & 15 & 12 & 16 & 21 & 32 & 30 & 36 & 41 \\
\hline
\end{tabular}

\begin{tabular}{rrrrrrrrrr|}
\hline$k$ & 12 & 13 & 14 & 15 & 16 & 17 & 18 & 19 & 20 \\
$H_{1}(k)$ & 38 & 42 & 46 & 50 & 64 & 59 & 63 & 67 & 71 \\
$H_{2}(k)$ & 45 & 50 & 54 & 59 & 65 & 69 & 74 & 78 & 83 \\
\hline
\end{tabular}

Finally, we remark that when $k$ is equal to 4 or 8 , the conclusion of Theorem 1.6 may be strengthened in the manner presented below. We briefly present details of the associated proof when $k=4$, but leave the case $k=8$ as an exercise for the reader. 
THEOREM 1.7. Suppose that $\phi_{2}$ is an integral quadratic polynomial with positive leading coefficient. Let $t$ be a non-negative integer.

(i) For almost all natural numbers $n$ satisfying $\phi_{2}(n) \equiv r(\bmod 16)$, for some $r$ with $1 \leq r \leq 8+t$, one has that $\phi_{2}(n)$ is the sum of $8+t$ biquadrates.

(ii) For almost all natural numbers $n$ satisfying $\phi_{2}(n) \equiv r(\bmod 32)$, for some $r$ with $1 \leq r \leq 26+t$, one has that $\phi_{2}(n)$ is the sum of $26+t$ eighth powers.

Throughout, the letters $\varepsilon$ and $\eta$ will denote sufficiently small positive numbers. We take $P$ to be the basic parameter, a large real number depending at most on $\varepsilon, \eta$ and any coefficients of implicit polynomials if necessary. We use $\ll$ and $\gg$ to denote Vinogradov's well-known notation, implicit constants depending at most on $\varepsilon, \eta$ and implicit polynomials. Sometimes we make use of vector notation. For example, the expression $\left(c_{1}, \ldots, c_{t}\right)$ is abbreviated to c. Also we write $[x]$ for the greatest integer not exceeding $x$, and $\lceil x\rceil$ for the least integer $y$ with $y \geq x$. Summations start at 1 unless indicated otherwise. In an effort to simplify our analysis, we adopt the following convention concerning the parameter $\varepsilon$. Whenever $\varepsilon$ appears in a statement, we assert that for each $\varepsilon>0$ the statement holds for sufficiently large values of the main parameter. Note that the "value" of $\varepsilon$ may consequently change from statement to statement, and hence also the dependence of implicit constants on $\varepsilon$.

We thank the referee for illuminating comments.

\section{Problems with a block of four cubes}

We begin our investigation of sums of mixed powers by establishing Theorems 1.1 and 1.2. Here we require some familiar estimates for mean values involving mixed powers which we summarise in the following lemma. Here and elsewhere, we write $e(z)$ for $e^{2 \pi i z}$.

Lemma 2.1. Suppose that $X$ is a large real number. When $1 \leq i \leq 3$, let $\phi_{i}$ denote an integral polynomial with positive leading coefficient of degree $d_{i}$, let $\kappa_{i}$ be a fixed positive real number, let $\mathscr{A}_{i} \subseteq \mathrm{N}$, write $X_{i}=X^{1 / d_{i}}$ and $A_{i}=\operatorname{card}\left(\mathscr{A}_{i} \cap\left[1, \kappa_{i} X_{i}\right]\right)$, and define

$$
\Theta_{i}(\alpha ; X)=\sum_{x \in \mathscr{A}_{i} \cap\left[1, \kappa_{i} X_{i}\right]} e\left(\alpha \phi_{i}(x)\right) .
$$

Then provided that $d_{i} \geq 2(i=1,2,3)$, one has

$$
\int_{0}^{1}\left|\Theta_{1}(\alpha ; X) \Theta_{2}(\alpha ; X) \Theta_{3}(\alpha ; X)\right|^{2} d \alpha \ll X^{\varepsilon}\left(A_{2}^{2} A_{3}^{2}+A_{1}\left(A_{3}^{2}+A_{2} A_{3}\right)\right) .
$$


Proof. By orthogonality, the mean value on the left hand side of (2.1) is equal to the number of solutions of the diophantine equation

$$
\sum_{i=1}^{3} \phi_{i}\left(x_{i}\right)=\sum_{i=1}^{3} \phi_{i}\left(y_{i}\right)
$$

with $x_{i}, y_{i} \in \mathscr{A}_{i} \cap\left[1, \kappa_{i} X_{i}\right](i=1,2,3)$. On observing that the polynomials $\phi_{i}(x)-\phi_{i}(y)$ are divisible by $x-y$ for $i=1,2,3$, the desired conclusion is immediate on separating out the diagonal terms, and employing an elementary estimate for the divisor function.

Corollary 2.2. When $d_{1}=2$ and $d_{2}^{-1}+d_{3}^{-1} \leq 1 / 2$, one has

$$
\int_{0}^{1}\left|\Theta_{1}(\alpha ; X) \Theta_{2}(\alpha ; X) \Theta_{3}(\alpha ; X)\right|^{2} d \alpha \ll X^{1 / d_{1}+1 / d_{2}+1 / d_{3}+\varepsilon} .
$$

Proof. Note that $A_{i} \ll X_{i}^{1 / d_{i}}$ and apply Lemma 2.1.

Our basic tool in the proofs of Theorems 1.1 and 1.2 is a certain mean value estimate for a block of four cubic exponential sums. In order to describe this fundamental estimate, we require some notation. When $X$ and $Y$ are positive numbers, we denote the set of $Y$-smooth numbers not exceeding $X$ by

$$
\mathscr{A}(X, Y)=\{n \in[1, X] \cap Z: p \text { prime, } p \mid n \Rightarrow p \leq Y\} .
$$

We take $P$ to be a large real number, write $\eta$ for a sufficiently small positive number depending at most on $\varepsilon$, and consider a real number $R$ with $P^{\eta / 2}<$ $R \leq P^{\eta}$. We write $Q=P^{6 / 7}, Y=P^{1 / 7}$, and define the generating functions

$$
f(\alpha ; p)=\sum_{\substack{P<x \leq 2 P \\ p \nmid x}} e\left(\alpha x^{3}\right), \quad g(\alpha)=\sum_{Q<y \leq 2 Q} e\left(\alpha y^{3}\right), \quad h(\alpha)=\sum_{z \in \mathscr{A}(Q, R)} e\left(\alpha z^{3}\right) .
$$

We then define, as our block of four cubic exponential sums,

$$
\mathscr{F}(\alpha)=\sum_{\substack{Y<p \leq 2 Y \\ p \equiv 2(\bmod 3)}} f(\alpha ; p) g\left(\alpha p^{3}\right) h\left(\alpha p^{3}\right)^{2},
$$

where the summation is over prime numbers.

In order to facilitate our application of the circle method, we define a generic Hardy-Littlewood dissection as follows. When $X$ is a real number with $1 \leq$ $X \leq P$, we define the set of major $\operatorname{arcs} \mathfrak{M}(X)$ to be the union of the intervals

$$
\mathfrak{M}(q, a)=\left\{\alpha \in[0,1):|q \alpha-a| \leq X P^{-3}\right\},
$$


with $0 \leq a \leq q \leq X$ and $(a, q)=1$. We define the corresponding set of minor arcs by $\mathfrak{m}(X)=[0,1) \backslash \mathfrak{M}(X)$. For brevity, it is convenient to write also

$$
\mathfrak{M}=\mathfrak{M}\left(P^{3 / 4}\right), \quad \mathfrak{m}=\mathfrak{m}\left(P^{3 / 4}\right),
$$

and, with $L=(\log P)^{1 / 100}$,

$$
\mathfrak{N}=\mathfrak{M}(L), \quad \mathfrak{n}=\mathfrak{m}(L) .
$$

Also, when $X$ is a positive number, we write

$$
\mathscr{R}(X)=\mathfrak{M}(2 X) \backslash \mathfrak{M}(X) .
$$

For the purposes of our exposition here, it suffices to consider the major arc approximations to $f(\alpha ; p)$ and $g\left(\alpha p^{3}\right)$. Thus, we define

$$
S(q, a)=\sum_{r=1}^{q} e\left(a r^{3} / q\right), \quad S(q, a, p)=S(q, a)-p^{-1} S\left(q, a p^{3}\right),
$$

and

$$
v(\beta)=\int_{P}^{2 P} e\left(\beta \gamma^{3}\right) d \gamma, \quad w(\beta)=\int_{Q}^{2 Q} e\left(\beta \gamma^{3}\right) d \gamma .
$$

Next define the functions $f_{p}^{*}$ and $g_{p}^{*}$ for $\alpha \in[0,1)$ by taking

$f_{p}^{*}(\alpha)=q^{-1} S(q, a, p) v\left(\alpha-\frac{a}{q}\right), \quad g_{p}^{*}(\alpha)=q^{-1} S\left(q, a p^{3}\right) w\left(p^{3}\left(\alpha-\frac{a}{q}\right)\right)$,

when $\alpha \in \mathfrak{M}(q, a) \subseteq \mathfrak{M}(P)$, and by taking each of these functions to be zero otherwise. Finally, we write

$$
\mathscr{F}_{1}(\alpha)=\sum_{\substack{Y<p \leq 2 Y \\ p \equiv 2(\bmod 3)}} f_{p}^{*}(\alpha) g_{p}^{*}(\alpha) h\left(\alpha p^{3}\right)^{2} .
$$

The crucial mean value estimates stemming from our block of four cubes may be summarised as follows.

Lemma 2.3. One has

$$
\int_{\mathfrak{m}}|\mathscr{F}(\alpha)|^{2} d \alpha \ll Y^{2} Q^{6} P^{-19 / 14}
$$

and

$$
\int_{\mathfrak{M}}\left|\mathscr{F}(\alpha)-\mathscr{F}_{1}(\alpha)\right|^{2} d \alpha \ll Y^{2} Q^{6} P^{-19 / 14}
$$

Proof. This is Lemma 3.2 of Brüdern, Kawada and Wooley [2]. 
Lemma 2.4. Suppose that $X$ is a real number with $1 \leq X \leq Q$. Then

$$
\int_{\mathfrak{M}_{(X)}}\left|\mathscr{F}_{1}(\alpha)\right| d \alpha \ll X^{\varepsilon} Y Q^{3} P^{-2}(\log Y)^{-1} .
$$

Proof. This is Lemma 3.3 of Brüdern, Kawada and Wooley [2].

Finally, we augment our stockpile of exponential sums by writing, for each natural number $k$,

$$
P_{k}=P^{3 / k} \quad \text { and } \quad f_{k}(\alpha)=\sum_{P_{k}<x \leq 2 P_{k}} e\left(\alpha x^{k}\right)
$$

Properly equipped at last, we launch our proof of Theorem 1.1.

Proof of Theorem 1.1. Let $\phi_{2} \in \mathrm{Q}[t]$ be an integral quadratic polynomial with positive leading coefficient. We take

$$
6 P^{3}=\phi_{2}(N)
$$

and denote by $\mathscr{Z}_{2}(N)$ the set of integers $n$ with $N<n \leq 2 N$ for which the diophantine equation

$$
\phi_{2}(n)=x_{1}^{3}+x_{2}^{3}+x_{3}^{3}+x_{4}^{3}+x_{5}^{3}+x_{6}^{6}
$$

has no solution in positive integers $x_{1}, \ldots, x_{6}$. We aim to show that $\operatorname{card}\left(\mathscr{Z}_{2}(N)\right) \ll N^{37 / 42+\varepsilon}$, and from this the conclusion of Theorem 1.1 follows by summing over dyadic intervals.

Write

$$
K_{2}(\alpha)=\sum_{n \in \mathscr{Z}_{2}(N)} e\left(\alpha \phi_{2}(n)\right)
$$

Then it follows from the definition of $\mathscr{Z}_{2}(N)$ that

$$
\int_{0}^{1} \mathscr{F}(\alpha) f_{3}(\alpha) f_{6}(\alpha) K_{2}(-\alpha) d \alpha=0 .
$$

On recalling Theorem 4.1 of Vaughan [12] for the purpose of analysing the behaviour of $f_{3}(\alpha)$ and $f_{6}(\alpha)$ on the set $\mathfrak{M}(q, a) \subseteq \mathfrak{N}$, the argument of the proof of Lemma 2.1 of Brüdern, Kawada and Wooley [2] is readily modified to provide in this instance the lower bound, uniformly for $5 P^{3} \leq m \leq 64 P^{3}$,

$$
\int_{\mathfrak{X}} \mathscr{F}(\alpha) f_{3}(\alpha) f_{6}(\alpha) e(-\alpha m) d \alpha \gg Y Q^{3} P^{-2}(\log P)^{-1} f_{3}(0) f_{6}(0) .
$$


Thus, on writing

$$
\mathscr{H}(\alpha)=f_{3}(\alpha) f_{6}(\alpha) K_{2}(-\alpha)
$$

we deduce from (2.6), (2.7) and (2.8) that

$$
\left|\int_{\mathfrak{n}} \mathscr{F}(\alpha) \mathscr{H}(\alpha) d \alpha\right| \gg Y Q^{3} P^{-2}(\log P)^{-1} \mathscr{H}(0) .
$$

Next we analyse the contribution arising from the set $\mathfrak{n \cap M}$. By the methods of Chapters 2 and 4 of Vaughan [12] (see, for example, equations (2.7), (2.8) and (3.11) of Brüdern, Kawada and Wooley [2]), one has for $X \leq P^{3 / 4}$ the estimate

$$
\sup _{\alpha \in \mathscr{R}(X)}\left|f_{3}(\alpha)\right| \ll P X^{-1 / 3}
$$

and hence it follows from Lemma 2.4 that for $X \leq P^{3 / 4}$, one has

$$
\begin{aligned}
\int_{\mathscr{R}(X)}\left|\mathscr{F}_{1}(\alpha) \mathscr{H}(\alpha)\right| d \alpha & \ll \mathscr{H}(0) X^{-1 / 3} \int_{\mathfrak{M}(2 X)}\left|\mathscr{F}_{1}(\alpha)\right| d \alpha \\
& \ll Y Q^{3} P^{-2} X^{-1 / 4}(\log Y)^{-1} \mathscr{H}(0) .
\end{aligned}
$$

On summing over $X=2^{l} L \leq P^{3 / 4}$ with $l \geq 0$, we obtain the upper bound

$$
\int_{\mathfrak{n} \cap \mathfrak{M}}\left|\mathscr{F}_{1}(\alpha) \mathscr{H}(\alpha)\right| d \alpha \ll Y Q^{3} P^{-2} L^{-1 / 4}(\log Y)^{-1} \mathscr{H}(0) .
$$

On recalling (2.9), we may conclude thus far that

$$
\begin{aligned}
\int_{\mathfrak{m}}|\mathscr{F}(\alpha) \mathscr{H}(\alpha)| d \alpha+\int_{\mathfrak{M}}\left|\left(\mathscr{F}(\alpha)-\mathscr{F}_{1}(\alpha)\right) \mathscr{H}(\alpha)\right| d \alpha \\
\\
\gg Y Q^{3} P^{-2}(\log Y)^{-1} \mathscr{H}(0) .
\end{aligned}
$$

By applying Schwarz's inequality to (2.10), and applying Lemma 2.3, we find that

$$
\begin{aligned}
& Q^{3} Y P^{-2}(\log Y)^{-1} \mathscr{H}(0) \\
& \quad \ll\left(\int_{\mathfrak{m}}|\mathscr{F}(\alpha)|^{2} d \alpha+\int_{\mathfrak{M}}\left|\mathscr{F}(\alpha)-\mathscr{F}_{1}(\alpha)\right|^{2} d \alpha\right)^{1 / 2}\left(\int_{0}^{1}|\mathscr{H}(\alpha)|^{2} d \alpha\right)^{1 / 2} \\
& \quad \ll\left(Y^{2} Q^{6} P^{-19 / 14}\right)^{1 / 2}\left(\int_{0}^{1}|\mathscr{H}(\alpha)|^{2} d \alpha\right)^{1 / 2} .
\end{aligned}
$$


Consequently, it follows from the corollary to Lemma 2.1 that

$$
\mathscr{H}(0) \ll P^{37 / 28}(\log P)\left(P^{3+\varepsilon}\right)^{1 / 2} \ll P^{79 / 28+\varepsilon},
$$

whence by (2.4), (2.5) and (2.8), we may conclude that

$$
\operatorname{card}\left(\mathscr{Z}_{2}(N)\right) \ll P^{79 / 28+\varepsilon}\left(P_{3} P_{6}\right)^{-1} \ll N^{37 / 42+\varepsilon} .
$$

The conclusion of Theorem 1.1 follows immediately.

The argument of the proof of Theorem 1.1 is readily adapted to tackle that of Theorem 1.2.

Proof of Theorem 1.2. We dispose of all the cases of the theorem simultaneously. Let $\phi_{i} \in \mathrm{Q}[t]$ denote an integral polynomial of degree $i$ with positive leading coefficient. When $i=2$ or 3 , we denote by $\mathscr{Z}_{i}^{l, k}(N)$ the set of all integers $n$ with $N<n \leq 2 N$ for which the diophantine equation

$$
\phi_{i}(n)=x_{1}^{3}+x_{2}^{3}+x_{3}^{3}+x_{4}^{3}+y^{l}+z^{k}
$$

has no solution in positive integers $x_{1}, \ldots, x_{4}, y, z$. We aim to show that, for appropriate choices of $(i, l, k)$, one has $\operatorname{card}\left(\mathscr{Z}_{i}^{l, k}(N)\right)=o(N)$, whence the conclusions of Theorem 1.2 will follow immediately by summing over dyadic intervals.

Define the exponential sum $K_{i}(\alpha)=K_{i}^{l, k}(\alpha)$ by

$$
K_{i}(\alpha)=\sum_{n \in \mathscr{C}_{i}^{l, k}(N)} e\left(\alpha \phi_{i}(n)\right) .
$$

Also, define the parameter $P$ by means of the relation

$$
6 P^{3}=\phi_{i}(N),
$$

and define the exponential sums $f_{j}(\alpha)$ as in (2.4). We then put

$$
\mathscr{H}(\alpha)=f_{l}(\alpha) f_{k}(\alpha) K_{i}(-\alpha) .
$$

Applying the arguments of the second paragraph of the proof of Theorem 1.1 above, and making use once more of Theorem 4.1 of Vaughan [12], we again obtain the lower bound (2.9). On the other hand, by Weyl's inequality (see Lemma 2.4 of Vaughan [12]) together with Lemma 2.8, Theorem 4.1 and Lemmata 4.3-4.5 of Vaughan [12], one has for $X \leq P^{3 / 4}$ the estimate

$$
\sup _{\alpha \in \Re(X)}\left|f_{l}(\alpha)\right| \ll P_{l} X^{\varepsilon-\left(l 2^{l}\right)^{-1}} .
$$


Thus we may apply the argument of the third paragraph of the proof of Theorem 1.1 to conclude that the upper bound (2.11) holds. Temporarily writing $Z_{i}^{l, k}=\operatorname{card}\left(\mathscr{Z}_{i}^{l, k}(N)\right)$, in the interest of clarity, and recalling (2.12) and (2.14), we therefore deduce from Lemma 2.1 that when $i=2$ and $2 \leq l \leq k$, one has $f_{l}(0) f_{k}(0) Z_{2}^{l, k}=\mathscr{H}(0) \ll P^{37 / 28+\varepsilon}\left(Z_{2}^{l, k}\left(P^{6 / k}+P^{3 / k+3 / l}\right)+P^{6 / k+6 / l}\right)^{1 / 2}$,

and when $i=3$ and $k \geq 3$, similarly,

$$
\begin{aligned}
f_{2}(0) f_{k}(0) Z_{3}^{2, k} & =\mathscr{H}(0) \\
& \ll P^{37 / 28+\varepsilon}\left(P^{3 / 2}\left(Z_{3}^{2, k} P^{3 / k}+P^{6 / k}\right)+\left(Z_{3}^{2, k}\right)^{2} P^{6 / k}\right)^{1 / 2} .
\end{aligned}
$$

A modest computation consequently leads from (2.4) and (2.13) to the estimates

$$
\begin{gathered}
\operatorname{card}\left(\mathscr{Z}_{2}^{3, k}(N)\right) \ll N^{23 / 21-2 / k+\varepsilon}+N^{37 / 42+\varepsilon}, \quad \operatorname{card}\left(\mathscr{Z}_{2}^{4,4}(N)\right) \ll N^{37 / 42+\varepsilon}, \\
\operatorname{card}\left(\mathscr{Z}_{2}^{5,5}(N)\right) \ll N^{101 / 105+\varepsilon}, \quad \operatorname{card}\left(\mathscr{Z}_{3}^{2,6}(N)\right) \ll N^{9 / 14+\varepsilon} .
\end{gathered}
$$

The conclusions of Theorem 1.2, with explicit estimates for the associated exceptional sets, follow immediately.

\section{Problems with a block of three cubes and a $k$ th power}

As indicated in the introduction, it is possible to modify the argument underlying the proofs of Theorems 1.1 and 1.2 so as to replace the block $\mathscr{F}(\alpha)$, of four cubic exponential sums, by a new block of three cubic exponential sums together with an exponential sum over a fourth, fifth or sixth power. We concentrate on the case with three cubes and a sixth power, since this provides a model for the treatment of the remaining, easier cases. We require an estimate from Brüdern and Wooley [6] in order to handle the mean value estimates which arise, and this forces us to introduce further notation.

By and large we adopt the same notation as that which we employed in $\S 2$. It is convenient, however, to recycle the latter notation by now writing

$$
Q=P^{7 / 8}, \quad Y=P^{1 / 8},
$$

and in addition defining the exponential sums

$$
\begin{aligned}
& F(\alpha)=\sum_{P<x \leq 2 P} e\left(\alpha x^{3}\right), \quad b(\alpha)=\sum_{y \in \mathscr{A}(\sqrt{P}, R)} e\left(\alpha y^{6}\right),
\end{aligned}
$$

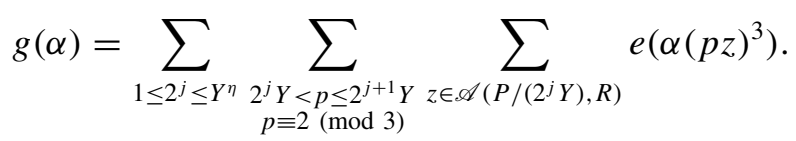


We then define the block $\mathscr{G}(\alpha)$ of exponential sums central to our subsequent argument by

$$
\mathscr{G}(\alpha)=F(\alpha) g(\alpha)^{2} b(\alpha) .
$$

It is the block $\mathscr{G}(\alpha)$ which plays a role in this section similar to that played in $\S 2$ by the block $\mathscr{F}(\alpha)$, defined in (2.2).

Lemma 3.1. Suppose that $X$ is a real number with $1 \leq X \leq P$. Then there is a fixed positive number $\tau$ with the property that

$$
\int_{\mathfrak{m}(X)}|\mathscr{G}(\alpha)|^{2} d \alpha \ll P^{4} X^{-\tau} .
$$

Proof. This is an immediate consequence of Theorem 4 of Brüdern and Wooley [6].

It is expedient to make use of a sharper version of the corollary to Lemma 2.1 in the pruning argument which occurs in our endgame analysis.

Lemma 3.2. Suppose that $X$ is a large real number. Let $\phi$ be an integral quadratic polynomial with positive leading coefficient. Let $\kappa$ be a fixed positive real number and let $\mathscr{A} \subseteq \mathrm{N} \cap\left[1, \kappa X^{1 / 2}\right]$. Define

$$
F_{j}(\alpha)=\sum_{y \leq X^{1 / j}} e\left(\alpha y^{j}\right), \quad G(\alpha)=\sum_{n \in \mathscr{A}} e(\alpha \phi(n)) .
$$

Then

$$
\int_{0}^{1}\left|G(\alpha) F_{4}(\alpha)^{2}\right|^{2} d \alpha \ll X(\log X)^{\varepsilon}
$$

and

$$
\int_{0}^{1}\left|G(\alpha) F_{3}(\alpha) F_{6}(\alpha)\right|^{2} d \alpha \ll X(\log X)^{\varepsilon} .
$$

Proof. This consequence of the work of Tenenbaum [9], Hooley [8] and Hall and Tenenbaum [7] is Lemma 3.1 of Brüdern, Kawada and Wooley [4].

An assault on the proof of Theorem 1.3 is now possible in easy stages.

ProOf OF THEOREM 1.3. We again dispose of all the cases simultaneously. Let $\phi_{i}$ denote an integral polynomial of degree $i$ with positive leading coefficient. When $i=2,3,4$ or 6 , we now denote by $\mathscr{Z}_{i}^{l, k}(N)$ the set of all integers $n$ with $N<n \leq \sqrt[6]{2} N$ for which the diophantine equation

$$
\phi_{i}(n)=x_{1}^{3}+x_{2}^{3}+x_{3}^{3}+x_{4}^{6}+y^{l}+z^{k}
$$


has no solution in positive integers $x_{1}, \ldots, x_{4}, y, z$. In the cases under consideration, we have that $\{i, k, l\}$ is one of $\{2,3,6\}$ or $\{2,4,4\}$, in the obvious sense. We aim to show that for such appropriate choices of $(i, k, l)$, one has $\operatorname{card}\left(\mathscr{Z}_{i}^{l, k}(N)\right)=o(N)$, whence the conclusions of Theorem 1.3 follow by summing over dyadic intervals.

Define the parameter $P$ and the exponential sums $K_{i}(\alpha)$ and $\mathscr{H}(\alpha)$ as in (2.12)-(2.14). Then arguing as in the proof of Lemma 2.1 of Brüdern, Kawada and Wooley [2] (see also the treatment of the major arcs $\mathfrak{N}$ in the proof of Lemma 2.1 of Brüdern, Kawada and Wooley [5]), one finds that uniformly for $4 P^{3} \leq m \leq 18 P^{3}$, one has the estimate

$$
\int_{\mathfrak{R}} \mathscr{G}(\alpha) f_{l}(\alpha) f_{k}(\alpha) e(-\alpha m) d \alpha \gg \sqrt{P} f_{l}(0) f_{k}(0) .
$$

Consequently, an argument akin to that yielding (2.9) on this occasion leads to the lower bound

$$
\left|\int_{\mathfrak{n}} \mathscr{G}(\alpha) \mathscr{H}(\alpha) d \alpha\right| \gg \sqrt{P} \mathscr{H}(0) .
$$

We take $\omega=10^{-4}$, and write $\mathfrak{B}=\mathfrak{M}\left(P^{\omega}\right)$ and $\mathfrak{p}=[0,1) \backslash \mathfrak{P}$. Then by the corollary to Lemma 2.1 , it follows that the upper bound

$$
\int_{0}^{1}|\mathscr{H}(\alpha)|^{2} d \alpha \ll P^{3+\varepsilon}
$$

holds in all cases under consideration. Consequently, an application of Schwarz's inequality in combination with Lemma 3.1 reveals that

$$
\begin{aligned}
\int_{\mathfrak{p}}|\mathscr{G}(\alpha) \mathscr{H}(\alpha)| d \alpha & \leq\left(\int_{\mathfrak{p}}|\mathscr{G}(\alpha)|^{2} d \alpha\right)^{1 / 2}\left(\int_{0}^{1}|\mathscr{H}(\alpha)|^{2} d \alpha\right)^{1 / 2} \\
& \ll P^{7 / 2-\sigma}
\end{aligned}
$$

for a suitable positive number $\sigma$.

In case (a) of Theorem 1.3, we have $i=2$, and an application of Schwarz's inequality yields

$$
\begin{aligned}
\int_{\mathfrak{n} \cap \Re_{\beta}}|\mathscr{G}(\alpha) \mathscr{H}(\alpha)| d \alpha & \\
& \ll\left(\int_{\mathfrak{n}}|\mathscr{G}(\alpha)|^{2} d \alpha\right)^{1 / 2}\left(\int_{0}^{1}\left|K_{2}(\alpha) f_{l}(\alpha) f_{k}(\alpha)\right|^{2} d \alpha\right)^{1 / 2} .
\end{aligned}
$$


We may apply Lemma 3.1 to the first integral on the right hand side, and Lemma 3.2 to the second. In this way we obtain the estimate

$$
\int_{\mathfrak{n} \cap \Re \mathfrak{B}}|\mathscr{G}(\alpha) \mathscr{H}(\alpha)| d \alpha \ll P^{7 / 2}(\log P)^{-\sigma},
$$

for a suitable positive number $\sigma$.

The bound (3.3) holds in all other cases of Theorem 1.3 as well, as we now demonstrate. The estimates all depend on the mean value

$$
\mathscr{K}=\int_{0}^{1}\left|f_{2}(\alpha) g(\alpha) b(\alpha)\right|^{2} d \alpha,
$$

for which, by considering the underlying diophantine equation, Lemma 3.2 yields the bound

$$
\mathscr{K} \ll P^{3}(\log P)^{\varepsilon} .
$$

In case (b), we have $i=3, l=2, k=6$, and Hölder's inequality produces

$$
\begin{aligned}
\int_{\mathfrak{n} \cap \mathfrak{\beta}}|\mathscr{G}(\alpha) \mathscr{H}(\alpha)| d \alpha \ll & \mathscr{K}^{1 / 2}\left(\int_{0}^{1}|g(\alpha)|^{8} d \alpha\right)^{1 / 8}\left(\int_{\mathfrak{B}}\left|f_{6}(\alpha)\right|^{8} d \alpha\right)^{1 / 8} \\
& \times\left(\int_{0}^{1}\left|K_{3}(\alpha)\right|^{12} d \alpha\right)^{1 / 12}\left(\int_{\mathfrak{n} \cap \mathfrak{R}}|F(\alpha)|^{6} d \alpha\right)^{1 / 6} .
\end{aligned}
$$

On considering the underlying diophantine equation and invoking Theorem 2 of Vaughan [10], one finds that

$$
\int_{0}^{1}|g(\alpha)|^{8} d \alpha \ll P^{5}
$$

The methods of Chapter 2 of Vaughan [12] will readily confirm the bound

$$
\int_{0}^{1}\left|K_{3}(\alpha)\right|^{12} d \alpha \leq \int_{0}^{1}\left|\sum_{n \leq 2 N} e\left(\alpha \phi_{3}(n)\right)\right|^{12} d \alpha \ll P^{9} .
$$

Finally, by the methods of Section 4.4 of Vaughan [12] (see, in particular, Lemma 4.9 and the proof of Theorem 4.4), one readily establishes that

$$
\int_{\mathfrak{B}}\left|f_{6}(\alpha)\right|^{8} d \alpha \ll P \quad \text { and } \quad \int_{\mathfrak{n} \cap \mathfrak{R}}|F(\alpha)|^{6} d \alpha \ll P^{3} L^{\varepsilon-2 / 3} \text {. }
$$

Collecting together the above estimates, we obtain (3.3) in case (b). Case (d) is quite similar. Here $i=6, l=2, k=3$, and we again use Hölder's inequality 
and the trivial bound $\left|K_{6}(\alpha)\right| \leq K_{6}(0) \ll \sqrt{P}$ to show that

$$
\begin{aligned}
& \int_{\mathfrak{n} \cap \Re_{\beta}}|\mathscr{G}(\alpha) \mathscr{H}(\alpha)| d \alpha \\
& \ll K_{6}(0) \mathscr{K}^{1 / 2}\left(\int_{0}^{1}|g(\alpha)|^{8} d \alpha\right)^{1 / 8}\left(\int_{\mathfrak{n} \cap \Re_{\mathfrak{\beta}}}\left|f_{3}(\alpha)\right|^{16 / 3} d \alpha\right)^{3 / 16} \\
& \times\left(\int_{\mathfrak{n} \cap \Re \beta}|F(\alpha)|^{16 / 3} d \alpha\right)^{3 / 16} .
\end{aligned}
$$

Here we note that again by the methods of Section 4.4 of Vaughan [12], one has

$$
\int_{\mathfrak{u} \cap \Re \mathfrak{\beta}}|F(\alpha)|^{16 / 3} d \alpha \ll P^{7 / 3} L^{\varepsilon-4 / 9},
$$

and the same estimate holds with $F$ replaced by $f_{3}$ (since, of course, the generating functions $F$ and $f_{3}$ are identical). Now (3.3) follows as in case (b).

In case (c) we have $i=4, l=2, k=4$. We bound $K_{4}(\alpha)$ trivially, and use Hölder's inequality in the form

$$
\begin{aligned}
& \int_{\mathfrak{n} \cap \Re}|\mathscr{G}(\alpha) \mathscr{H}(\alpha)| d \alpha \\
& \ll K_{4}(0) \mathscr{K}^{1 / 2}\left(\int_{0}^{1}|g(\alpha)|^{8} d \alpha\right)^{1 / 8}\left(\int_{\mathfrak{n} \cap \Re \mathfrak{\beta}}|F(\alpha)|^{16 / 3} d \alpha\right)^{3 / 16} \\
& \times\left(\int_{\mathfrak{\Re}}\left|f_{4}(\alpha)\right|^{16 / 3} d \alpha\right)^{3 / 16} .
\end{aligned}
$$

Now, in order to confirm (3.3) in this final case, it suffices to add to the previous mean values the estimate

$$
\int_{\mathfrak{B}}\left|f_{4}(\alpha)\right|^{16 / 3} d \alpha \ll\left(P^{3 / 4}\right)^{16 / 3-4}=P,
$$

which is again a straightforward consequence of the methods of Section 4.4 of Vaughan [12], since 16/3 exceeds 5.

Having now established (3.3) in all cases, we combine this bound with (3.1) and (3.2) to infer that $\sqrt{P} \mathscr{H}(0) \ll \int_{\mathfrak{p}}|\mathscr{G}(\alpha) \mathscr{H}(\alpha)| d \alpha+\int_{\mathfrak{n} \cap \Re \beta}|\mathscr{G}(\alpha) \mathscr{H}(\alpha)| d \alpha \ll P^{7 / 2}(\log P)^{-\sigma}$, whence, in view of (2.4), (2.13) and (2.14), the upper bound $\operatorname{card}\left(\mathscr{Z}_{i}^{l, k}(N)\right) \ll$ $N(\log N)^{-\sigma}$ follows in all cases under consideration. This completes the proof of Theorem 1.3. 


\section{Sums of four cubes and a square}

The existence of a square in a given representation problem permits a powerful application of Weyl's inequality for the associated exponential sum. The difficulties to be negotiated in the prosecution of our methods are then shifted to the problem of pruning back to a sufficiently narrow set of major arcs, as will become evident in our proof of Theorem 1.4 below. In what follows, it is convenient to discard the notation of the previous two sections and begin anew. It is useful to record for future use the following mean value estimate.

Lemma 4.1. Let $U(X)$ denote the number of solutions of the diophantine equation

$$
x_{1}^{3}-x_{2}^{3}=y_{1}^{3}+y_{2}^{3}-y_{3}^{3}-y_{4}^{3},
$$

with $1 \leq x_{i} \leq 2 X(i=1,2)$ and $y_{j} \in \mathscr{A}\left(X, X^{\eta}\right)(1 \leq j \leq 4)$. Then provided that $\eta>0$ is sufficiently small, one has

$$
U(X) \ll X^{13 / 4-2 \eta} .
$$

Proof. The conclusion of the lemma follows from Theorem 1.2 of Wooley [21].

Proof of Theorem 1.4. Let $\phi_{3} \in \mathrm{Q}[t]$ denote an integral cubic polynomial with positive leading coefficient. We denote by $\mathscr{Z}(N)$ the set of all integers $n$ with $N / 2<n \leq N$ for which the diophantine equation

$$
\phi_{3}(n)=x_{1}^{3}+x_{2}^{3}+x_{3}^{3}+x_{4}^{3}+y^{2}
$$

has no solution in positive integers $x_{1}, \ldots, x_{4}, y$. We aim to show that $\operatorname{card}(\mathscr{Z}(N))=o(N)$, and just as in previous discussions, the conclusion of Theorem 1.4 will follow by summing over dyadic intervals.

When $k=2$ and 3, define the parameter $P_{k}$ by means of the relation $P_{k}=\left(\phi_{3}(N)\right)^{1 / k}$, and then define

$$
F_{k}(\alpha)=\sum_{x \leq P_{k}} e\left(\alpha x^{k}\right) \quad \text { and } \quad f_{k}(\alpha)=\sum_{y \in \mathscr{A}\left(P_{k}, P_{k}^{n}\right)} e\left(\alpha y^{k}\right),
$$

where $\eta$ is a sufficiently small positive number. Write also

$$
K(\alpha)=\sum_{n \in \mathscr{Z}(N)} e\left(\alpha \phi_{3}(n)\right) .
$$

When $1 \leq Q \leq N^{3 / 2}$, we define the major $\operatorname{arcs} \mathfrak{M}(Q)$ to be the union of the intervals

$$
\mathfrak{M}(q, a ; Q)=\left\{\alpha \in[0,1):|q \alpha-a| \leq Q N^{-3}\right\},
$$


with $0 \leq a \leq q \leq Q$ and $(a, q)=1$. For the sake of concision, we write

$$
\mathfrak{M}=\mathfrak{M}\left(N^{4 / 3}\right) \quad \text { and } \quad \mathfrak{N}=\mathfrak{M}(L),
$$

where $L=(\log N)^{1 / 100}$. We also write $\mathfrak{m}=[0,1) \backslash \mathfrak{M}$ and $\mathfrak{n}=[0,1) \backslash \mathfrak{R}$.

Observe first that the definition of $\mathscr{Z}(N)$ implies the identity

$$
\int_{0}^{1} F_{2}(\alpha) F_{3}(\alpha)^{2} f_{3}(\alpha)^{2} K(-\alpha) d \alpha=0 .
$$

Next, in a manner similar to the treatments applied in previous examples, the methods of Vaughan [11], Vaughan [12, §4.4], and Vaughan and Wooley [13] provide the lower bound

$$
\int_{\Re} F_{2}(\alpha) F_{3}(\alpha)^{2} f_{3}(\alpha)^{2} e(-\alpha m) d \alpha \gg P_{2} P_{3},
$$

uniformly for $\phi_{3}(N / 2)<m \leq \phi_{3}(N)$. Thus it follows from (4.1) that

$$
N^{5 / 2} \operatorname{card}(\mathscr{Z}(N)) \ll \int_{\mathfrak{n}}\left|F_{2}(\alpha) F_{3}(\alpha)^{2} f_{3}(\alpha)^{2} K(\alpha)\right| d \alpha .
$$

We next remove the minor arcs $m$ from the integral on the right hand side. By Weyl's inequality (see, for example, Lemma 2.4 of Vaughan [12]), one has

$$
\sup _{\alpha \in \mathfrak{m}}\left|F_{2}(\alpha)\right| \ll P_{2}^{1+\varepsilon} N^{-2 / 3} \ll N^{5 / 6+\varepsilon} .
$$

Consequently, on recalling Lemma 4.1 and Hua's Lemma (see Lemma 2.5 of Vaughan [12]), and considering the underlying diophantine equations, one deduces that

$$
\begin{gathered}
\int_{\mathfrak{m}}\left|F_{2}(\alpha) F_{3}(\alpha)^{2} f_{3}(\alpha)^{2} K(\alpha)\right| d \alpha \\
\ll\left(\sup _{\alpha \in \mathfrak{m}}\left|F_{2}(\alpha)\right|\right)\left(\int_{0}^{1}\left|F_{3}(\alpha) f_{3}(\alpha)^{2}\right|^{2} d \alpha\right)^{1 / 2} \\
\quad \times\left(\int_{0}^{1}\left|F_{3}(\alpha)\right|^{4} d \alpha\right)^{1 / 4}\left(\int_{0}^{1}|K(\alpha)|^{4} d \alpha\right)^{1 / 4} \\
\ll N^{5 / 6+\varepsilon}\left(P_{3}^{13 / 4-\tau}\right)^{1 / 2}\left(P_{3}^{2+\varepsilon}\right)^{1 / 4}\left(N^{2+\varepsilon}\right)^{1 / 4},
\end{gathered}
$$

for a suitable positive number $\tau$. We thus obtain the upper bound

$$
\int_{\mathfrak{m}}\left|F_{2}(\alpha) F_{3}(\alpha)^{2} f_{3}(\alpha)^{2} K(\alpha)\right| d \alpha \ll N^{83 / 24} \ll P_{2} P_{3} N^{23 / 24} .
$$


In order to proceed further we recall some notation. When $k=2$ or 3 , define the generating functions

$$
S_{k}(q, a)=\sum_{r=1}^{q} e\left(a r^{k} / q\right), \quad v_{k}(\beta)=\int_{0}^{P_{k}} e\left(\beta \gamma^{k}\right) d \gamma
$$

and define the functions $F_{k}^{*}(\alpha)$ for $\alpha \in \mathfrak{M}\left(q, a ; N^{4 / 3}\right) \subseteq \mathfrak{M}$ by taking

$$
F_{k}^{*}(\alpha)=q^{-1} S_{k}(q, a) v_{k}(\alpha-a / q)
$$

Then by Theorem 4.1 of Vaughan [12], for $k=2$ or 3,

$$
\sup _{\alpha \in \mathfrak{M}}\left|F_{k}(\alpha)-F_{k}^{*}(\alpha)\right| \ll N^{2 / 3+\varepsilon},
$$

and by Lemma 4.6 of Vaughan [12], one also has

$$
\left|F_{k}^{*}(\alpha)\right| \ll P_{k}\left(q+N^{3}|q \alpha-a|\right)^{-1 / k}
$$

A comparison of (4.5) and (4.3) reveals that the treatment of the minor arcs $\mathrm{m}$ is readily modified to show that

$$
\int_{\mathfrak{M}}\left|\left(F_{2}(\alpha)-F_{2}^{*}(\alpha)\right) F_{3}(\alpha)^{2} f_{3}(\alpha)^{2} K(\alpha)\right| d \alpha \ll P_{2} P_{3} N^{23 / 24}
$$

We observe next that in view of (4.6), it follows from Lemma 2 of Brüdern [1] that

$$
\int_{\mathfrak{M}}\left|F_{2}^{*}(\alpha) K(\alpha)\right|^{2} d \alpha \ll N^{7 / 3+\varepsilon}
$$

Thus an application of Schwarz's inequality combined with (4.5) and Lemma 4.1 leads to the estimate

$$
\begin{aligned}
\int_{\mathfrak{M}}\left|\left(F_{3}(\alpha)-F_{3}^{*}(\alpha)\right) F_{2}^{*}(\alpha) F_{3}(\alpha) f_{3}(\alpha)^{2} K(\alpha)\right| d \alpha \\
\ll N^{2 / 3+\varepsilon}\left(\int_{\mathfrak{M}}\left|F_{2}^{*}(\alpha) K(\alpha)\right|^{2} d \alpha\right)^{1 / 2}\left(\int_{0}^{1}\left|F_{3}(\alpha) f_{3}(\alpha)^{2}\right|^{2} d \alpha\right)^{1 / 2} \\
\ll N^{2 / 3+\varepsilon}\left(N^{7 / 3+\varepsilon}\right)^{1 / 2}\left(P_{3}^{13 / 4-\tau}\right)^{1 / 2} \ll P_{2} P_{3} N^{23 / 24}
\end{aligned}
$$

Here again $\tau$ is used to denote a suitable positive real number. 
The next two steps in the main argument require some additional mean values which we now collect. On considering the underlying diophantine equations, the methods of Chapter 2 of Vaughan [12] suffice to confirm the bounds

$$
\int_{0}^{1}\left|f_{3}(\alpha)\right|^{10} d \alpha \ll P_{3}^{7} \quad \text { and } \quad \int_{0}^{1}|K(\alpha)|^{10} d \alpha \ll P_{3}^{7} .
$$

Similarly, by Hua's Lemma (see Lemma 2.5 of Vaughan [12]), one has

$$
\int_{0}^{1}\left|f_{3}(\alpha)\right|^{8} d \alpha \ll P_{3}^{5+\varepsilon}
$$

A straightforward application of the Hardy-Littlewood method, using Lemma 4.9 of Vaughan [12], demonstrates that

$$
\int_{\mathfrak{M}}\left|F_{3}^{*}(\alpha)\right|^{4} d \alpha \ll P_{3}^{1+\varepsilon}
$$

and in much the same way we confirm the bound

$$
\int_{\mathfrak{M \cap n}}\left|F_{3}^{*}(\alpha)\right|^{30 / 7} d \alpha \ll P_{3}^{9 / 7} L^{\varepsilon-2 / 21} .
$$

Finally, as an elementary consequence of (4.6), one has

$$
\int_{\mathfrak{M}}\left|F_{2}^{*}(\alpha)\right|^{30 / 7} d \alpha \ll P_{2}^{30 / 7} N^{-3}
$$

By Hölder's inequality, the estimates (4.5) and (4.8), and the above mean values, we deduce that

$$
\int_{\mathfrak{M}}\left|\left(F_{3}(\alpha)-F_{3}^{*}(\alpha)\right) F_{2}^{*}(\alpha) F_{3}^{*}(\alpha) f_{3}(\alpha)^{2} K(\alpha)\right| d \alpha
$$

$$
\begin{aligned}
& \ll N^{2 / 3+\varepsilon}\left(\int_{\mathfrak{M}}\left|F_{2}^{*}(\alpha) K(\alpha)\right|^{2} d \alpha\right)^{1 / 2}\left(\int_{0}^{1}\left|f_{3}(\alpha)\right|^{8} d \alpha\right)^{1 / 4}\left(\int_{\mathfrak{M}}\left|F_{3}^{*}(\alpha)\right|^{4} d \alpha\right)^{1 / 4} \\
& \ll N^{2 / 3+\varepsilon}\left(N^{7 / 3+\varepsilon}\right)^{1 / 2}\left(P_{3}^{5+\varepsilon}\right)^{1 / 4}\left(P_{3}^{1+\varepsilon}\right)^{1 / 4} \ll P_{2} P_{3} N^{5 / 6+\varepsilon} .
\end{aligned}
$$

On collecting together (4.2), (4.4), (4.7), (4.9) and (4.13), we may conclude thus far that

$$
N^{5 / 2} \operatorname{card}(\mathscr{Z}(N)) \ll \int_{\mathfrak{M} \cap \mathfrak{n}}\left|F_{2}^{*}(\alpha) F_{3}^{*}(\alpha)^{2} f_{3}(\alpha)^{2} K(\alpha)\right| d \alpha+O\left(P_{2} P_{3} N^{23 / 24}\right) .
$$


An application of Hölder's inequality, making use of the bounds (4.10), (4.11) and (4.12), shows that

$$
\begin{aligned}
\int_{\mathfrak{M} \cap \mathfrak{n}}\left|F_{2}^{*}(\alpha) F_{3}^{*}(\alpha)^{2} f_{3}(\alpha)^{2} K(\alpha)\right| d \alpha & \\
\ll & \left(\int_{\mathfrak{M}}\left|F_{2}^{*}(\alpha)\right|^{30 / 7} d \alpha\right)^{7 / 30}\left(\int_{\mathfrak{M} \cap \mathfrak{n}}\left|F_{3}^{*}(\alpha)\right|^{30 / 7} d \alpha\right)^{7 / 15} \\
& \times\left(\int_{0}^{1}\left|f_{3}(\alpha)\right|^{10} d \alpha\right)^{1 / 5}\left(\int_{0}^{1}|K(\alpha)|^{10} d \alpha\right)^{1 / 10} \\
\ll & N^{7 / 2} L^{\varepsilon-2 / 45} .
\end{aligned}
$$

Thus we may conclude from (4.14) that card $(\mathscr{Z}(N)) \ll N L^{\varepsilon-2 / 45}$, and the conclusion of the theorem follows on summing over dyadic intervals.

By working harder on the major arcs, it is possible to refine the above argument to obtain the estimate $\operatorname{card}(\mathscr{Z}(N)) \ll N^{23 / 24}$. In accordance with the opening comments of this paper, we leave it to the reader to provide the details of such a refinement. The inquisitive readers possessing an unexpected abundance of leisure time may also care to entertain themselves by establishing that almost all values of a given integral quadratic polynomial are the sum of a square, a cube, a biquadrate, a fifth power, a sixth power and a seventh power.

\section{Waring's problem for larger exponents in general}

Moving in this section from the more exotic problems involving mixed sums of powers, to the more classical Waring's problem for $k$ th powers, our objective is the proof of Theorem 1.5 for polynomial sequences of degree exceeding 2 . We do not aim here for estimates possessing the sharpest error terms, preferring at this point concision over precision. In the next section, we satisfy our desire for sharp conclusions with a more detailed account of quadratic sequences. We begin here by recording some notation. Let $\phi(t)=\phi_{l}(t)$ be an integral polynomial of degree $l \geq 2$ with positive leading coefficient, and suppose that $k$ is sufficiently large. We take $N$ to be a large real number, and write

$$
P=\phi_{l}(N)^{1 / k}, \quad L=(\log P)^{1 / 100} \quad \text { and } \quad R=P^{\eta},
$$

where $\eta>0$ is supposed to be sufficiently small. We remark that the first of these relations implies that $P \asymp N^{l / k}$. We then write

$$
H(\alpha)=\sum_{x \leq P} e\left(\alpha x^{k}\right) \quad \text { and } \quad h(\alpha)=\sum_{x \in \mathscr{A}(P, R)} e\left(\alpha x^{k}\right) .
$$


Before advancing to the main body of our argument, we pause to record some auxiliary mean value estimates. Here it is convenient to introduce some notation for Hardy-Littlewood dissections. When $Q$ is a positive number, we define the set of major $\operatorname{arcs} \mathfrak{M}(Q)$ to be the union of the intervals

$$
\mathfrak{M}(q, a ; Q)=\left\{\alpha \in[0,1):|q \alpha-a| \leq Q P^{-k}\right\},
$$

with $0 \leq a \leq q \leq Q$ and $(a, q)=1$. For the sake of brevity, we then write

$$
\mathfrak{M}=\mathfrak{M}(P), \quad \mathfrak{N}=\mathfrak{M}(L), \quad \mathfrak{m}=[0,1) \backslash \mathfrak{M}, \quad \mathfrak{n}=[0,1) \backslash \mathfrak{N} .
$$

Lemma 5.1. Define the natural numbers $m, t, u, v, w$ by

$$
\begin{gathered}
m=\left\lceil\left(\frac{1}{4}+\frac{1}{2} \log 2\right) k\right\rceil, \quad u=\left\lceil\frac{1}{2} k(1+1 / \sqrt{\log k})\right\rceil, \quad v=k, \\
t=\left\lceil\frac{1}{2} k(\log k+\log \log k+1)\right\rceil
\end{gathered}
$$

and

$$
w=\left\lceil\frac{1}{2}\left(1-\frac{2}{l}\right) k(\log k+\log \log k+1)\right\rceil+\left\lceil 3\left(1-\frac{2}{l}\right) k\right\rceil .
$$

Then we have

$$
\begin{aligned}
\int_{0}^{1}\left|H(\alpha)^{2} h(\alpha)^{2 m-2}\right| d \alpha & \ll P^{2 m-k / 2}, \\
\int_{0}^{1}|h(\alpha)|^{2(t+u)} d \alpha & \ll P^{2(t+u)-k}, \\
\int_{0}^{1}|h(\alpha)|^{2 v l} d \alpha & \ll P^{2 v l-k\left(1-2^{-l}\right)},
\end{aligned}
$$

and when $l>2$, one has also

$$
\int_{0}^{1}|h(\alpha)|^{2 w l /(l-2)} d \alpha \ll P^{2 w l /(l-2)-k} .
$$

Proof. We recall that the methods of Wooley [18] (see the conclusion of $\S 3$ of [18] and the corollary to Theorem 2.1 of [19]) provide the upper bounds

$$
\int_{0}^{1}\left|H(\alpha)^{2} h(\alpha)^{2 r-2}\right| d \alpha \ll P^{2 r-k+\Delta_{r}+\varepsilon},
$$

and

$$
\int_{0}^{1}|h(\alpha)|^{2 r} d \alpha \ll P^{2 r-k+\Delta_{r}+\varepsilon}
$$


where $\Delta_{r}$ is the positive number satisfying the equation

$$
\Delta_{r} e^{\Delta_{r} / k}=k e^{1-2 r / k} .
$$

In particular, of course, one has $\Delta_{r}<k e^{1-2 r / k}$. In addition, Theorem 1.1 of Wooley [20] shows that

$$
\sup _{\alpha \in \mathfrak{m}}|h(\alpha)| \ll P^{1-\rho(k)},
$$

where $\rho(k)^{-1}=k(\log k+O(\log \log k))$.

We note first that since $m>\left(\frac{1}{4}+\frac{1}{2} \log 2\right) k$, it follows from (5.5) and (5.7) that

$$
\int_{0}^{1}\left|H(\alpha)^{2} h(\alpha)^{2 m-2}\right| d \alpha \ll P^{2 m-k+\Delta_{m}+\varepsilon},
$$

where $\Delta_{m}$ satisfies the inequality $\Delta_{m} e^{\Delta_{m} / k}<\frac{1}{2} e^{1 / 2} k$. The latter inequality implies that $\Delta_{m}<k / 2$, and so (5.1) follows immediately. Also, since

$$
\Delta_{v l} \leq k e^{1-2 v l / k}=k e^{1-2 l}<k 2^{-l},
$$

we find from (5.6) that the estimate (5.3) also holds. Furthermore, since $w l /(l-2) \geq t+u$, possibly by employing the trivial estimate $|h(\alpha)| \leq P$, one finds that (5.4) is an immediate consequence of (5.2).

In view of the preceding remarks, we concentrate on establishing the bound (5.2). Here we apply the Hardy-Littlewood method. By (5.5) and (5.7), one has

$$
\int_{0}^{1}\left|H(\alpha)^{2} h(\alpha)^{2 t-2}\right| d \alpha \ll P^{2 t-k+\Delta_{t}+\varepsilon},
$$

where

$$
\Delta_{t}<k \exp (1-(\log k+\log \log k+1))=1 / \log k .
$$

Thus, on recalling (5.8), one finds that

$$
\begin{aligned}
\int_{\mathfrak{m}}\left|H(\alpha)^{2} h(\alpha)^{2(t+u)-2}\right| d \alpha & \leq\left(\sup _{\alpha \in \mathfrak{m}}|h(\alpha)|\right)^{2 u} \int_{0}^{1}\left|H(\alpha)^{2} h(\alpha)^{2 t-2}\right| d \alpha \\
& \ll P^{2(t+u)-k-\delta}
\end{aligned}
$$

where

$$
\delta=2 u \rho(k)-\frac{1}{\log k}=\frac{1}{(\log k)^{3 / 2}}+O\left(\frac{\log \log k}{(\log k)^{2}}\right)>0 .
$$


On the other hand, a familiar treatment of the major arcs $\mathfrak{M}$ (see, for example, $\S 5$ of Vaughan [11]) reveals that

$$
\int_{\mathfrak{M}}\left|H(\alpha)^{2} h(\alpha)^{2(t+u)-2}\right| d \alpha \ll P^{2(t+u)-k} .
$$

On combining (5.9) and (5.10), we deduce that

$$
\begin{aligned}
& \int_{0}^{1}\left|H(\alpha)^{2} h(\alpha)^{2(t+u)-2}\right| d \alpha \\
& \quad=\int_{\mathfrak{M}}\left|H(\alpha)^{2} h(\alpha)^{2(t+u)-2}\right| d \alpha+\int_{\mathfrak{m}}\left|H(\alpha)^{2} h(\alpha)^{2(t+u)-2}\right| d \alpha \ll P^{2(t+u)-k},
\end{aligned}
$$

so that on considering the underlying diophantine equation, we find that

$$
\int_{0}^{1}|h(\alpha)|^{2(t+u)} d \alpha \leq \int_{0}^{1}\left|H(\alpha)^{2} h(\alpha)^{2(t+u)-2}\right| d \alpha \ll P^{2(t+u)-k} .
$$

This completes the proof of the lemma.

We are now equipped to describe the proof of Theorem 1.5 when $d=l>2$. Write

$$
s_{1}=\lceil(1-1 / l) k(\log k+\log \log k+20)\rceil,
$$

and put $s=s_{1}+2$. We denote by $\mathscr{Z}(N)$ the set of integers $n$ with $N / 2<n \leq N$ for which the diophantine equation (1.1) has no solution in positive integers $x_{1}, \ldots, x_{s}$. We aim to show that $\operatorname{card}(\mathscr{Z}(N))=o(N)$, whence as usual the desired conclusion follows by summing over dyadic intervals.

Define the exponential sum

$$
K_{l}(\alpha)=\sum_{n \in \mathscr{Z}(N)} e\left(\alpha \phi_{l}(n)\right) .
$$

Then it follows from the definition of $\mathscr{Z}(N)$ that

$$
\int_{0}^{1} H(\alpha)^{2} h(\alpha)^{s_{1}} K_{l}(-\alpha) d \alpha=0 .
$$

In a manner similar to, but simpler than, the treatments of previous sections, the methods of Vaughan [11], Vaughan [12, §4.4], and Vaughan and Wooley [13] lead to the lower bound

$$
\int_{\mathfrak{R}} H(\alpha)^{2} h(\alpha)^{s_{1}} e(-\alpha n) d \alpha \gg P^{s-k},
$$


uniformly for $2^{-1-l} P^{k}<n \leq P^{k}$, provided only that $s \geq 4 k$. Thus, when $k$ is sufficiently large, it follows from (5.12) that

$$
P^{s-k} \operatorname{card}(\mathscr{Z}(N)) \ll \int_{\mathfrak{n}}\left|H(\alpha)^{2} h(\alpha)^{s_{1}} K_{l}(\alpha)\right| d \alpha .
$$

Standard pruning arguments based on the above cited methods establish the estimate

$$
\int_{\mathfrak{n} \cap \mathfrak{M}}\left|H(\alpha)^{2} h(\alpha)^{s_{1}}\right| d \alpha \ll P^{s-k} L^{-1 / k},
$$

whence by a trivial estimate for $K_{l}(\alpha)$, we deduce from (5.13) that

$$
\int_{\mathfrak{m}}\left|H(\alpha)^{2} h(\alpha)^{s_{1}} K_{l}(\alpha)\right| d \alpha \gg P^{s-k} \operatorname{card}(\mathscr{Z}(N)) .
$$

We next apply Lemma 4.1 of Brüdern, Kawada and Wooley [4] with

$$
\mathscr{F}(\alpha)=h(\alpha)^{t+u}, \quad \mathscr{G}(\alpha)=h(\alpha)^{v}, \quad \mathscr{H}(\alpha)=h(\alpha)^{w} .
$$

On noting that $P^{k} \asymp N^{l}$, we find that the conclusions (5.2)-(5.4) of Lemma 5.1 above imply the validity of the hypotheses (4.1)-(4.3) of [4, Lemma 4.1]. Since the latter lemma provides the upper bound

$$
\int_{0}^{1}\left|K_{l}(\alpha) \mathscr{F}(\alpha) \mathscr{G}(\alpha)^{2} \mathscr{H}(\alpha)\right| d \alpha \ll N^{-l+1+\varepsilon} \mathscr{F}(0) \mathscr{G}(0)^{2} \mathscr{H}(0),
$$

on recalling the definition (5.11) of $s_{1}$, and applying the trivial bound $|h(\alpha)| \leq$ $P$, we conclude that

$$
\int_{0}^{1}\left|K_{l}(\alpha) h(\alpha)^{s_{1}}\right| d \alpha \ll P^{s_{1}} N^{-l+1+\varepsilon} .
$$

But by Weyl's inequality (see, for example, Lemma 2.4 of Vaughan [12]), one has

$$
\sup _{\alpha \in \mathfrak{m}}|H(\alpha)| \ll P^{1-2^{1-k}+\varepsilon},
$$

and thus it follows that

$$
\begin{aligned}
\int_{\mathfrak{m}}\left|H(\alpha)^{2} h(\alpha)^{s_{1}} K_{l}(\alpha)\right| d \alpha & \leq\left(\sup _{\alpha \in \mathfrak{m}}|H(\alpha)|\right)^{2} \int_{0}^{1}\left|K_{l}(\alpha) h(\alpha)^{s_{1}}\right| d \alpha \\
& \ll P^{s-2^{1-k}} N^{1-l} .
\end{aligned}
$$

On recalling once again that $N^{l} \asymp P^{k}$, we conclude from (5.14) that

$$
P^{s} N^{-l} \operatorname{card}(\mathscr{Z}(N)) \ll P^{s-2^{1-k}} N^{1-l},
$$


whence $\operatorname{card}(\mathscr{Z}(N)) \ll N^{1-2^{1-k} l / k}$. On summing over dyadic intervals, we therefore find that $G_{l}^{+}(k) \leq s$, and the conclusion of Theorem 1.5 follows whenever $d=l>2$.

\section{Waring's problem for larger exponents and quadratic sequences}

The proof of Theorem 1.5 is completed with only slight additional effort, and since the proof of Theorem 1.6 involves only a modicum of extra exertion, we take care of both tasks together. We remark, however, that for reasons depending on local solubility considerations, our argument so far as the proof of Theorem 1.6 is concerned for $k=4$ and 8 is better handled by way of the proof of Theorem 1.7. In addition, the exponent $k=6$ requires a slightly more subtle approach, a topic that we discuss briefly in due course.

We adopt the same notation as that employed in $\S 5$, save that $l$ is now set equal to 2. Let $m$ be any integer satisfying the condition that the estimate (5.1) holds. In particular, when $k$ is large, it follows from Lemma 5.1 that

$$
m=\left\lceil\left(\frac{1}{4}+\frac{1}{2} \log 2\right) k\right\rceil
$$

is permissible. For smaller values of $k$, one may read from the tables of Vaughan and Wooley [16], [17] that the values of $m$ recorded in the table below are permissible. Also, let $a$ be any integer satisfying the condition that the upper bound

$$
\int_{\mathfrak{m}}\left|H(\alpha)^{2} h(\alpha)^{2 a-2}\right| d \alpha \ll P^{2 a-k-\tau}
$$

holds for some positive number $\tau$. Thus, when $k$ is large, one finds from the estimate (5.9) that

$$
a=\left\lceil\frac{1}{2} k(\log k+\log \log k+2+1 / \sqrt{\log k})\right\rceil+2
$$

is permissible. For smaller values of $k$, one may again verify from the tables and methods of Vaughan and Wooley [16], [17] that the values of $a$ recorded in the table below suffice (excluding the case $k=6$ ).

\begin{tabular}{|ccccccccc|}
\hline$k$ & 5 & 6 & 7 & 8 & 9 & 10 & 11 & 12 \\
$m$ & 3 & 4 & 4 & 5 & 5 & 6 & 7 & 7 \\
$a$ & 9 & 12 & 17 & 21 & 25 & 30 & 34 & 38 \\
\hline
\end{tabular}




\begin{tabular}{|ccccccccc|}
\hline$k$ & 13 & 14 & 15 & 16 & 17 & 18 & 19 & 20 \\
$m$ & 8 & 8 & 9 & 10 & 10 & 11 & 11 & 12 \\
$a$ & 42 & 46 & 50 & 55 & 59 & 63 & 67 & 71 \\
\hline
\end{tabular}

We now set $s=m+a, s_{1}=s-2$, and define $\mathscr{Z}(N)$ and $K_{l}(\alpha)$ as in $\S 5$. The argument of $\S 5$ now leads us, without further alteration, to the conclusion (5.14). In order to estimate the left hand side of (5.14), we apply Schwarz's inequality to obtain

$$
\int_{\mathfrak{m}}\left|K_{2}(\alpha) H(\alpha)^{2} h(\alpha)^{s_{1}}\right| d \alpha \leq \mathscr{I}_{1}^{1 / 2} \mathscr{I}_{2}^{1 / 2},
$$

where

$$
\mathscr{I}_{1}=\int_{0}^{1}\left|K_{2}(\alpha)^{2} H(\alpha)^{2} h(\alpha)^{2 m-2}\right| d \alpha \quad \text { and } \quad \mathscr{I}_{2}=\int_{\mathfrak{m}}\left|H(\alpha)^{2} h(\alpha)^{2 a-2}\right| d \alpha .
$$

On considering the underlying diophantine equation, we find that $\mathscr{I}_{1}$ is bounded above by the number of integral solutions of the equation

$$
\phi_{2}\left(n_{1}\right)-\phi_{2}\left(n_{2}\right)=\sum_{i=1}^{m}\left(x_{i}^{k}-y_{i}^{k}\right),
$$

with $n_{j} \in \mathscr{Z}(N)(j=1,2), x_{1}, y_{1} \leq P$ and $x_{i}, y_{i} \in \mathscr{A}(P, R)(2 \leq i \leq m)$. On recalling that the polynomial $\phi_{2}$ is quadratic, we find that an elementary estimate for the divisor function shows that for a fixed choice of $\mathbf{x}$ and $\mathbf{y}$ for which the right hand side of (6.5) is non-zero, one has that the number of solutions $n_{1}, n_{2}$ of (6.5) counted by $\mathscr{I}_{1}$ is $O\left(N^{\varepsilon}\right)$. When the right hand side of (6.5) is zero, meanwhile, one necessarily has $n_{1}=n_{2}$. Consequently, on considering the underlying diophantine equation, one obtains

$$
\mathscr{I}_{1} \ll N \int_{0}^{1}\left|H(\alpha)^{2} h(\alpha)^{2 m-2}\right| d \alpha+N^{\varepsilon} P^{2 m},
$$

whence by the assumed estimate (5.1) and the relation $P^{k} \asymp N^{2}$,

$$
\mathscr{I}_{1} \ll N P^{2 m-k / 2}+N^{\varepsilon} P^{2 m} \ll P^{2 m+\varepsilon} .
$$

In view of (6.2), therefore, we deduce from (6.4) that

$$
\begin{aligned}
\int_{\mathfrak{m}}\left|K_{2}(\alpha) H(\alpha)^{2} h(\alpha)^{s_{1}}\right| d \alpha & \ll\left(P^{2 a-k-\tau}\right)^{1 / 2}\left(P^{2 m+\varepsilon}\right)^{1 / 2} \\
& \ll P^{s-k / 2-\tau / 3} .
\end{aligned}
$$


On substituting (6.6) into (5.14), we arrive at the bound

$$
P^{s-k} \operatorname{card}(\mathscr{Z}(N)) \ll P^{s-k / 2-\tau / 3},
$$

whence the relation $P^{k} \asymp N^{2}$ reveals that

$$
\operatorname{card}(\mathscr{Z}(N)) \ll P^{k / 2-\tau / 3} \ll N^{1-\sigma},
$$

for a positive number $\sigma$. On summing over dyadic intervals, we therefore conclude that $G_{2}^{+}(k) \leq m+a$, and in view of (6.1), (6.3) and the entries in the above table, the proofs of Theorems 1.5 and 1.6 are now complete in the cases currently under consideration.

We return briefly to the topic of the exponent $k=6$. Here the estimate (6.2) is no longer available directly from the work of Vaughan and Wooley [16], [17]. In this case one must replace the occurrence of $h(\alpha)$ in (6.2) by the new exponential sum

$$
h(\alpha)=\sum_{\substack{P^{\theta} \leq M \leq P^{\theta+\tau} \\ M=2^{u} P^{\theta}}} \sum_{\substack{M<p \leq 2 M \\ p \equiv-1(\bmod 6)}} \sum_{\substack{y \in \mathscr{A}(P / M, R)\\}} e\left(\alpha(p y)^{6}\right),
$$

with a certain real number $\theta$ slightly smaller than 0.154543 , and with $\tau>0$ sufficiently small. The details can be found in Vaughan and Wooley [15], where the reader will discover that the desired estimate (6.2) follows from Lemma 6.5 of [15] following some modest pruning of the type implicit in Lemma 7.1 of that paper. With this adjustment, the argument described above again applies, though the treatment of the major arcs $\mathfrak{M}$ necessarily becomes somewhat more elaborate. We leave the verification of all the details of this argument as an entertaining exercise for the reader.

Finally, we remark that the only significant complications associated with the treatment of the cases $k=4$ and 8 concern the congruence conditions stemming from the prime 2 . When $k=8$, one must negotiate a congruence condition modulo 32, and when $k=4$, there is a congruence condition modulo 16. A brief discussion of this issue is contained in the following sketch of the proof of Theorem 1.7 (i).

Proof of TheOrem 1.7 (i). Let $\phi_{2}(n)$ be an integral quadratic polynomial with positive leading coefficient. We denote by $\mathscr{Z}(N)$ the set of all integers $n$ with $N / 2<n \leq N$ for which $\phi_{2}(n)$ satisfies the congruence condition in the statement of Theorem 1.7 (i), and yet the diophantine equation

$$
\phi_{2}(n)=x_{1}^{4}+x_{2}^{4}+\cdots+x_{8+t}^{4}
$$


has no solution in positive integers $x_{1}, \ldots x_{8+t}$. Write $P=\phi_{2}(N)^{1 / 4}$ and $L=(\log P)^{1 / 100}$, and define

$$
F(\alpha)=\sum_{x \leq P} e\left(\alpha x^{4}\right), \quad f(\alpha)=\sum_{x \in \mathscr{A}(P, R)} e\left(\alpha x^{4}\right) .
$$

Define the major arcs $\mathfrak{N}$ to be the union of the intervals

$$
\mathfrak{N}(q, a)=\left\{\alpha \in[0,1):|q \alpha-a| \leq L P^{-4}\right\},
$$

with $0 \leq a \leq q \leq L$ and $(a, q)=1$, and define $\mathfrak{n}=[0,1) \backslash \mathfrak{R}$.

The methods of Vaughan [11], as in the previous examples of this paper, show that uniformly for $P^{4} / 6 \leq m \leq 6 P^{4}$ with $m \equiv r(\bmod 16)$ and $1 \leq$ $r \leq 8+t$, one has the estimate

$$
\int_{\mathfrak{R}} F(\alpha)^{3} f(\alpha)^{5+t} e(-\alpha m) d \alpha \gg P^{4+t},
$$

and by the now familiar routine we deduce that

$$
\left|\int_{\mathfrak{n}} F(\alpha)^{3} f(\alpha)^{5+t} K_{2}(-\alpha) d \alpha\right| \gg P^{4+t} \operatorname{card}(\mathscr{Z}(N)) .
$$

On the other hand, on combining methods of Vaughan [11] and Wooley [18] with the conclusion of Lemma 3.2, one obtains by Schwarz's inequality

$$
\begin{aligned}
\int_{\mathfrak{n}}\left|F(\alpha)^{3} f(\alpha)^{5+t} K_{2}(\alpha)\right| d \alpha & \\
\leq & \left(\int_{0}^{1}\left|K_{2}(\alpha) F(\alpha)^{2}\right|^{2} d \alpha\right)^{1 / 2}\left(\int_{\mathfrak{n}}\left|F(\alpha) f(\alpha)^{5+t}\right|^{2} d \alpha\right)^{1 / 2} \\
& \ll\left(P^{4}(\log P)^{\varepsilon}\right)^{1 / 2}\left(P^{8+2 t}(\log P)^{-3 \sigma}\right)^{1 / 2},
\end{aligned}
$$

for a suitable positive number $\sigma$. Combining (6.7) and (6.8), we deduce that

$$
\operatorname{card}(\mathscr{Z}(N)) \ll P^{2}(\log P)^{-\sigma} \ll N(\log N)^{-\sigma},
$$

and thus the desired conclusion follows on summing over dyadic intervals.

\section{REFERENCES}

1. Brüdern, J., A problem in additive number theory, Math. Proc. Cambridge Philos. Soc. 103 (1988), 27-33.

2. Brüdern, J., Kawada, K. and Wooley, T. D., Additive representation in thin sequences, I: Waring's problem for cubes, Ann. Sci. École Norm. Sup. (4) 34 (2001), 471-501. 
3. Brüdern, J., Kawada, K. and Wooley, T. D., Additive representation in thin sequences, II: the binary Goldbach problem, Mathematika 47 (2000), 117-125.

4. Brüdern, J., Kawada, K. and Wooley, T. D., Additive representation in thin sequences, III: asymptotic formulae, Acta Arith. 100 (2001), 267-289.

5. Brüdern, J., Kawada, K. and Wooley, T. D., Additive representation in thin sequences, IV: lower bound methods, Quart. J. Math. Oxford 52 (2001), 423-436.

6. Brüdern, J. and Wooley, T. D., On Waring's problem: three cubes and a sixth power, Nagoya Math. J. 163 (2001), 13-53.

7. Hall, R. and Tenenbaum, G., Divisors, Cambridge University Press, Cambridge, 1988.

8. Hooley, C., On a new technique and its applications to the theory of numbers, Proc. London Math. Soc. (3) 38 (1979), 115-151.

9. Tenenbaum, G., Fonctions $\Delta$ de Hooley et applications, Sém. Theorie des Nombres, Paris 1984-85. Prog. Math. 63 (1986), 225-239.

10. Vaughan, R. C., On Waring's problem for cubes, J. Reine Angew. Math. 365 (1986), 122-170.

11. Vaughan, R. C., A new iterative method in Waring's problem, Acta Math. 162 (1989), 1-71.

12. Vaughan, R. C., The Hardy-Littlewood method, 2nd. ed., Cambridge University Press, Cambridge, 1997.

13. Vaughan, R. C. and Wooley, T. D., On Waring's problem: some refinements, Proc. London Math. Soc. (3) 63 (1991), 35-68.

14. Vaughan, R. C. and Wooley, T. D., Further improvements in Waring's problem, III: eighth powers, Philos. Trans. Roy. Soc. London Ser. A 345 (1993), 385-396.

15. Vaughan, R. C. and Wooley, T. D., Further improvements in Waring's problem, II: sixth powers, Duke Math. J. 76 (1994), 683-710.

16. Vaughan, R. C. and Wooley, T. D., Further improvements in Waring's problem, Acta Math. 174 (1995), 147-240.

17. Vaughan, R. C. and Wooley, T. D., Further improvements in Waring's problem, IV: higher powers, Acta Arith. 94 (2000), 203-285.

18. Wooley, T. D., Large improvements in Waring's problem, Ann. of Math. (2) 135 (1992), 131-164.

19. Wooley, T. D., The application of a new mean value theorem to the fractional parts of polynomials, Acta Arith. 65 (1993), 163-179.

20. Wooley, T. D., New estimates for smooth Weyl sums, J. London Math. Soc. (2) 51 (1995), $1-13$.

21. Wooley, T. D., Breaking classical convexity in Waring's problem: sums of cubes and quasidiagonal behaviour, Invent. Math. 122 (1995), 421-451.

MATHEMATISCHES INSTITUT A

UNIVERSITÄT STUTTGART

POSTFACH 801140

D-70511 STUTTGART

GERMANY

E-mail: bruedern@mathematik.uni-stuttgart.de
DEPARTMENT OF MATHEMATICS

FACULTY OF EDUCATION

IWATE UNIVERSITY

MORIOKA 020-8550

JAPAN

E-mail: kawada@iwate-u.ac.jp

DEPARTMENT OF MATHEMATICS

UNIVERSITY OF MICHIGAN

EAST HALL

525 EAST UNIVERSITY AVENUE

ANN ARBOR, MICHIGAN 48109-1109

USA

E-mail: wooley@math.lsa.umich.edu 